\title{
Valuación de activos intangibles con matemática difusa y su adecuación a normas contables españolas e internacionales
}

\author{
PAULINO E. MALLO; MARÍA ANTONIA ARTOLA; MARIANO MORETTINI; \\ MARCELO J. GALANTE; MARIANO E. PASCUAL y ADRIÁN R. BUSETTO \\ Grupo de Investigación Matemática Borrosa \\ Facultad de Ciencias Económicas y Sociales \\ UNIVERSIDAD NACIONAL DE MAR DEL PLATA, ARGENTINA \\ e-mail: mariano.morettini@gmail.com
}

\section{RESUMEN}

El presente trabajo tiene por objetivo plantear una alternativa de valuación de activos intangibles a la luz de la importancia creciente que los mismos han adquirido en los últimos tiempos en relación a los bienes físicos o materiales.

Específicamente, intentamos brindar una posibilidad de valuación de los bienes inmateriales a través de la utilización de la Matemática Difusa, y planteamos la compatibilidad de estas técnicas con los principios y requisitos de la información contable españoles e internacionales, a fin de que pueda incorporarse dicha valuación a los estados contables preparados por las empresas.

Palabras clave: Activos intangibles, Matemática Difusa, Contabilidad.

\section{Valuation of intangible assets with Fuzzy Mathematics and its adaptation to Spanish and International accounting standards}

\footnotetext{
ABSTRACT

The aim of this paper is to expose an alternative form of valuation of intangible assets according to their growing importance in the past years in comparison with physical goods.

Specifically, we intend to give a possibility of valuation of intangible goods using Fuzzy Mathematics, and we analyze the compatibility of these techniques with the Spanish and international accounting principles, in order to incorporate our valuation propose to the financial statements of enterprises.

Keywords: Intangible assets, Fuzzy Mathematics, Accounting.

Clasificación JEL: C0, D8, M4.
}

Artículo recibido en abril de 2008 y aceptado para su publicación en julio de 2008.

Artículo disponible en versión electrónica en la página www.revista-eea.net, ref. @26208. 


\section{PRESENTACIÓN E IMPORTANCIA DEL TEMA}

La realidad de las últimas décadas nos indica que la importancia que han adquirido los bienes intangibles como creadores de valor ha crecido a un ritmo vertiginoso, inclusive por encima de lo que han experimentado los bienes físicos.

Ejemplos de ello no escasean en ningún país y en ningún ramo de actividad. Podemos mencionar la necesidad de la Bolsa de Valores de Estados Unidos de crear un nuevo índice, el NASDAQ, que comprende a las empresas de alta generación de valor intangible, como las empresas de última tecnología en electrónica, informática, telecomunicaciones, biotecnología, entre otras, para que se presenten en forma independiente a las compañías tradicionales, cotizantes en el Dow Jones.

También podemos mencionar la marcada diferencia existente entre los valores contables de los bienes físicos de una compañía en relación a sus valores reales, que contemplan a los bienes intangibles de una manera más acabada que lo que lo hace la contabilidad. Y aquí podemos mencionar desde el clásico ejemplo del valor de la fórmula de la Coca Cola hasta el valor de un cuadro de Van Gogh o una silla diseñada por un artista de reconocimiento internacional.

Las obras de arte, entonces, son claros ejemplos de la importancia de los intangibles, puesto que su valor radica no en los bienes físicos que los involucran - pintura, madera, lienzo, metales - sino en que plasman el virtuosismo de sus creadores, y es ese bien intangible el que les da real valor a esos objetos.

Pero la importancia de estos bienes no es privativo de obras de arte o de empresas de alta tecnología, sino que se evidencia en toda clase de compañías y organizaciones humanas. Basta con analizar los gastos crecientes que todas las empresas efectúan en pos de acrecentar sus activos intangibles - como sus marcas, patentes, softwares, etc.- ya que los mismos están íntimamente vinculados con la generación de valor de las empresas mismas.

Una prueba más de la relevancia de este tema es el tratamiento que le dio el Internacional Accounting Standards Comitee en 1998 cuando redactó la NIC 38 y en 2004 cuando la modificó. Asimismo, y para el caso de España, la adopción del nuevo Plan General Contable a partir de 2008 presenta algunas modificaciones respecto del anterior, de 1990, y se adapta a la norma internacional respectiva.

Si bien las mencionadas normas no se apartan demasiado de la posición conservadora tradicional, han dejado vislumbrar una cierta flexibilización, buscando otorgar a los activos intangibles un papel más destacado en los estados contables que el que tradicionalmente tuvieron.

Es así como, por ejemplo, se permite, en ciertos casos, el reconocimiento contable de algunos intangibles autogenerados, además de los adquiridos. Por otra parte, la importancia de los activos intangibles se plasma en el hecho que se requiere informar sobre dichos activos, además de la valuación indicada en los estados contables, cierta información que pueda ser subjetiva y que no constituyen valuaciones monetarias referida a los intangibles, y que sea de utilidad para los distintos usuarios de los estados contables. 
En este trabajo presentamos los conceptos introductorios de la Matemática Difusa, plantearemos algunas alternativas de valuación de activos intangibles con dichas técnicas y analizaremos su cumplimiento de los requisitos de la información contable, según normas españolas e internacionales, a la vez de extraer conclusiones sobre su mejor cumplimiento de los objetivos de los estados contables.

\section{CONCEPTOS INTRODUCTORIOS DE MATEMÁTICA DIFUSA}

Las propuestas que presentaremos se basan en la utilización de la Matemática Difusa. Durante siglos la Matemática se basó en el principio aristotélico del tercero excluido, según el cual un elemento puede formar o no parte de un conjunto dado, negando una tercera posibilidad.

El surgimiento de la lógica multivaluada, a comienzos del siglo XX, admite todas las alternativas posibles entre los dos extremos aristotélicos, es decir, un elemento puede pertenecer a un conjunto en un grado dado. Así, y siguiendo el ejemplo utilizado por Lofti Zadeh ${ }^{1}$, en el conjunto de las mujeres bellas encontraremos a mujeres de distintas clases, las que tendrán un grado de pertenencia variable a dicho conjunto.

Basándose en esta nueva lógica nace la Matemática Borrosa, que trabaja con números borrosos.

Siguiendo a Kaufmann y Gil Aluja (1987), podemos decir que si consideramos una variable que puede tomar cualquier valor dentro del conjunto de los números reales, con la condición de que éste sea mayor o igual a un número al y menor o igual a $a_{2}$, diremos que el segmento $\mathrm{A}=\left[a_{1} ; a_{2}\right]$, siendo $a_{1} \leq a_{2}$ y ambos pertenecientes al conjunto de los números reales, es el intervalo de confianza relativo a la variable considerada.

Por su parte, y siguiendo a los mismos autores, un número borroso está formado por una secuencia finita o infinita de intervalos de confianza con las siguientes propiedades:

(a) Se afecta a cada intervalo de confianza un valor $\alpha \in[0,1]$, de tal manera que dos intervalos de confianza diferentes no pueden tener el mismo valor $\alpha$, al que se lo denomina "nivel de presunción" o "nivel de confianza";

(b) Los intervalos de confianza deben encajarse los unos con los otros, esto es, para cada intervalo de confianza de nivel $\alpha$, representado por $\mathrm{A} \alpha=\left[a_{1}(\alpha) ; a_{2}(\alpha)\right]$, se debe cumplir $\left(\alpha^{\prime}<\alpha\right) \Rightarrow\left(\mathrm{A} \alpha \supset \mathrm{A} \alpha^{\prime}\right)$;

(c) Existe un intervalo y sólo uno que puede reducirse a un real único.

${ }^{1}$ Zadeh, Lofti A.(1965). Pág. 338. 
Las formas de presentar a los números borrosos, según Kaufmann y Gil Aluja (1987) y Lazzari, Machado y Pérez (1998), entre otros, son:

(a) Asignar a cada nivel $\alpha$ un intervalo de confianza, es decir, $\forall \alpha \in[0,1]$ será $\mathrm{A} \alpha=$ $\left[a_{1}(\alpha) ; a_{2}(\alpha)\right]$

(b) Designar mediante $\mu(\mathrm{x})$ una función que representa los niveles del número borroso para cada valor de $\mathrm{x} \in \mathfrak{R}$.

Los números borrosos más utilizados son los triangulares $(\mathrm{NBT})^{2}$, que son, siguiendo a Lazzari, Machado y Pérez (1998), los números borrosos reales continuos tales que su función de pertenencia determina con el eje horizontal un triángulo. Su función de pertenencia es lineal a izquierda y derecha y se grafica como se muestra en la Figura 1.

FIGURA 1

Gráfico de NBT.

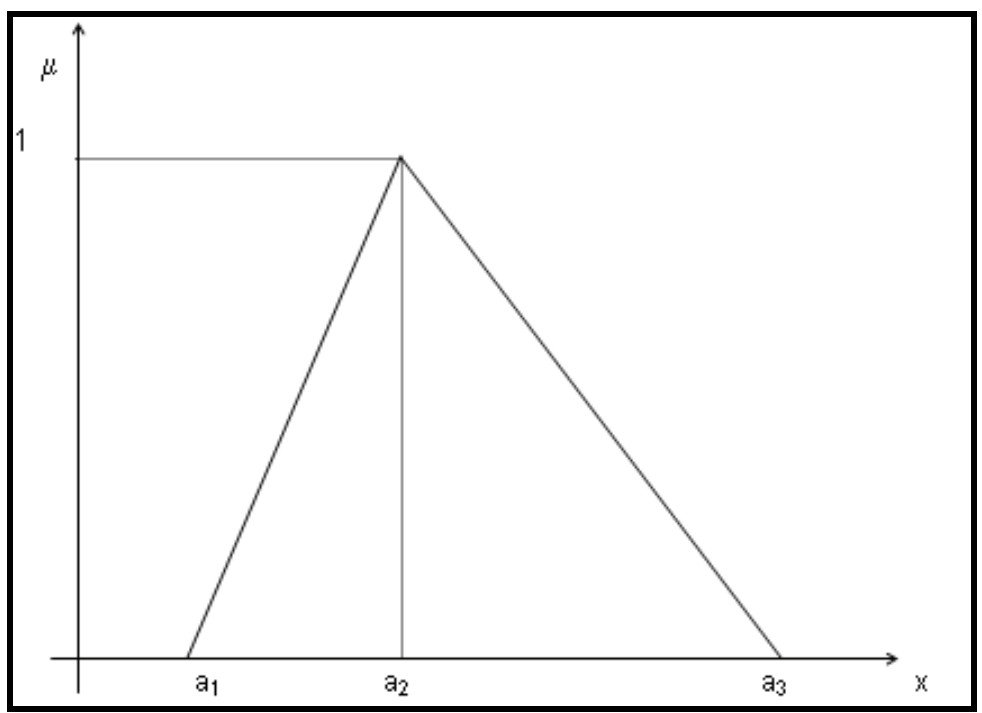

${ }^{2}$ Cabe aclarar que, si bien con una lógica distinta, la teoría de grafos aplicada a la planificación de proyectos (PERT y CPM) pueden considerarse un antecedente de los NBTs. 
De esta manera, la función de pertenencia es:

$$
\mu(\mathrm{x})= \begin{cases}0 & \text { si } \mathrm{x}<a_{1} \\ \frac{x-a_{1}}{a_{2}-a_{1}} & \text { si } a_{1} \leq \mathrm{x} \leq a_{2} \\ \frac{-x+a_{3}}{a_{3}-a_{2}} & \text { si } a_{2} \leq \mathrm{x} \leq a_{3} \\ 0 & \text { si } \mathrm{x}>a_{3}\end{cases}
$$

Por ejemplo, si se consulta a un experto acerca del valor que estima tendrá el tipo de cambio $€ / \mathrm{U} \$ S$ al $31 / 12 / 08$, por ejemplo, una respuesta podría ser: "se encontrará entre 1,50 $\left(a_{1}\right)$ y 1,80 $\left(a_{3}\right)$, pero el valor al que más confianza puede asignársele será $1,62\left(a_{2}\right)$ ".

A partir de estos datos se construye el NBT suponiendo una confianza creciente en forma lineal para los valores ubicados entre el mínimo y el más confiable y decreciente linealmente para los ubicados entre el más confiable y el máximo.

Para definir las operaciones aritméticas con números borrosos, seguiremos el método de los intervalos de confianza.

Así, para la adición de dos NBTs $\tilde{A}$ y $\tilde{B}$ cuyos intervalos de confianza son

$$
\mathrm{A} \alpha=\left[a_{1}(\alpha) ; a_{2}(\alpha)\right] \text { y } \mathrm{B} \alpha=\left[b_{1}(\alpha) ; b_{2}(\alpha)\right],
$$

la suma de ambos dará por resultado

$$
A \alpha(+) \mathrm{B} \alpha=\left[a_{1}(\alpha)+b_{1}(\alpha) ; a_{2}(\alpha)+b_{2}(\alpha)\right]
$$

Para una mayor comprensión procederemos a graficar dos NBTs y la suma de ambos en la Figura 2.

FIGURA 2

Gráfico de suma de NBTs.

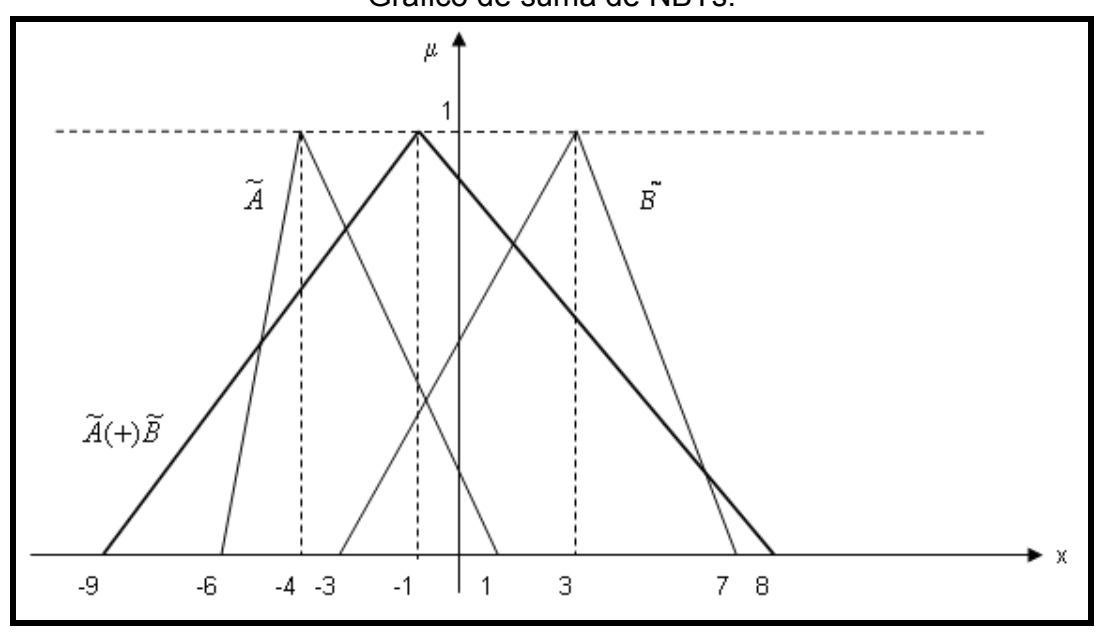


Si lo que deseamos es restar NBTs, procederemos de la siguiente manera:

$$
\mathrm{A} \alpha(-) \mathrm{B} \alpha=\left[a_{1}(\alpha)-b_{2}(\alpha) ; a_{2}(\alpha)-b_{1}(\alpha)\right]
$$

Para la multiplicación deben considerarse los mínimos y los máximos de los intervalos de confianza, de forma que el resultado será:

$$
\begin{gathered}
\mathrm{A} \alpha(.) \mathrm{B} \alpha=\left[\min \left(a_{1}(\alpha) \cdot b_{1}(\alpha), a_{1}(\alpha) \cdot b_{2}(\alpha), a_{2}(\alpha) \cdot b_{1}(\alpha), a_{2}(\alpha) \cdot b_{2}(\alpha)\right) ;\right. \\
\left.\operatorname{máx}\left(a_{1}(\alpha) \cdot b_{1}(\alpha), a_{1}(\alpha) \cdot b_{2}(\alpha), a_{2}(\alpha) \cdot b_{1}(\alpha), a_{2}(\alpha) \cdot b_{2}(\alpha)\right)\right]
\end{gathered}
$$

En forma análoga, para la división tendremos que:

$$
\begin{aligned}
\mathrm{A} \alpha(:) \mathrm{B} \alpha=\left[\min \left(a_{1}(\alpha): b_{1}(\alpha), a_{1}(\alpha): b_{2}(\alpha), a_{2}(\alpha): b_{1}(\alpha), a_{2}(\alpha): b_{2}(\alpha)\right) ;\right. \\
\left.\operatorname{máx}\left(a_{1}(\alpha): b_{1}(\alpha), a_{1}(\alpha): b_{2}(\alpha), a_{2}(\alpha): b_{1}(\alpha), a_{2}(\alpha): b_{2}(\alpha)\right)\right]
\end{aligned}
$$

La forma en que se graficarían estas operaciones es similar a la indicada en la Figura 2, por lo que nos remitimos a ella.

\section{LA INFORMACIÓN CONTABLE Y SUS USUARIOS}

Entre las variadas definiciones que se han dado de "Contabilidad", reproducimos la del autor argentino Enrique Fowler Newton: "la contabilidad, parte integrante del sistema de información de un ente, es la técnica de procesamiento de datos que permite obtener información sobre la composición y evolución del patrimonio de dicho ente, los bienes de propiedad de terceros en su poder y ciertas contingencias. Dicha información debería ser de utilidad para facilitar las decisiones de los administradores del ente y de los terceros que interactúan o pueden llegar a interactuar con él, así como para permitir una eficaz vigilancia sobre los recursos y obligaciones del ente"

Según el Marco para la Preparación y Presentación de Estados Financieros del Internacional Standard Accounting Comitee, los estados financieros tienen como destinatarios a sus potenciales usuarios, por ejemplo, posibles inversores, empleados, prestamistas, proveedores, acreedores, clientes, gobierno y público en general.

Dentro del Balance, los activos intangibles son los que más dificultades y controversias presentan a la hora de su valuación. Analizaremos, pues, las alternativas que existen a la hora de valuar y exponer el rubro sub examine a la luz de la información que deben contener los estados contables y la utilidad que ellos representan para los tomadores de decisiones.

Siguiendo a Juan Carlos Viegas y otros, "uno de los aspectos más relevantes que pueden llegar a caracterizar la importancia de los estados contables, es su capacidad para dar respuestas a aquellos que están vinculados o pretenden relacionarse con la entidad" ". A continuación analizan la importancia de la información contenida en los estados contables para el control de gestión, la financiación de la empresa, el valor de la empresa y el saneamiento financiero.

\footnotetext{
${ }^{3}$ Fowler Newton, Enrique (2005).

${ }^{4}$ Viegas, J.C.; Fronti, L.; Chaves, O.A. y Pahlen Acuña, J.M. (1997); página 30.
} 
Respecto del control de gestión, los estados contables presentan la medición de los resultados del ejercicio en relación con la inversión. En cuanto a la financiación de la empresa, brindan información sobre los pasivos tomados que deben cancelarse en el corto plazo, lo cual es de importancia no sólo para los accionistas sino también para los proveedores y acreedores de la entidad, tanto actuales como potenciales. Para inversiones de largo plazo los autores destacan la importancia de los estados contables proyectados. Sobre el valor de la empresa, los autores limitan la utilidad de los estados contables de cierre de ejercicio. Abonando esta posición, las Normas Internacionales de Contabilidad, en el Boletín $\mathrm{N}^{\mathrm{o}} 15$ del IASC, prescriben que no es objetivo de los estados financieros determinar el valor empresa" ${ }^{\text {. }}$. Destacan en este punto la importancia de los activos intangibles y, en menor medida, de las contingencias.

Por último, respecto del saneamiento financiero, los autores se refieren a la situación de empresas en crisis. Nuevamente aquí la utilidad de los estados contables es limitada, porque las decisiones se basan en proyecciones no incluidas en los estados contables.

Entre las limitaciones que Viegas y sus colegas advierten en los estados contables, citan a Kennedy y McMullen, para quienes los estados contables "son esencialmente informes provisionales y, por lo tanto, no pueden ser definitivos, porque la ganancia o pérdida real del negocio sólo puede determinarse cuando se vende o es liquidado"6.

Se destaca, a continuación, la opinión de Anthony ${ }^{7}$, para quien las principales limitaciones de los estados contables son: a) los estados contables sólo incluyen información que puede expresarse en forma monetaria, aunque las notas, que son parte integrante de los estados contables, los complementan salvando parte de esta limitación; b) los valores contables de los activos raramente son los de mercado, lo cual dependerá del modelo contable a utilizar; c) algunas valuaciones contables son estimativas por depender de acontecimientos futuros.

En un trabajo de 1974, el doctrinario Santiago Lazzati ${ }^{8}$ afirma que el objetivo de los estados contables es "brindar a sus usuarios información objetiva sobre la riqueza actual de la empresa y su evolución habida durante el último período (o últimos), con la finalidad de que ellos puedan tomar debidamente las decisiones que les competen"'.

Vemos que aparece en la definición de Lazzati una condición que es un principio general de la contabilidad: la objetividad. Cabe preguntarse si respetando rígidamente este principio se estaría cumpliendo con los objetivos de los estados contables.

\footnotetext{
${ }^{5}$ Viegas, J.C.; Fronti, L.; Chaves, O.A. y Pahlen Acuña, J.M. (1997); página 33.

${ }^{6}$ Kennedy y McMullen; Estados financieros; UTEHA; 1972; pp. 10 y 11; citados por Viegas, J.C.; Fronti, L.; Chaves, O.A. y Pahlen Acuña, J.M. (1997); página 34.

${ }^{7}$ Anthony, R.N.; La contabilidad en la administración de empresas; UTEHA; México; 1971; página 258; citado por Viegas, J.C.; Fronti, L.; Chaves, O.A. y Pahlen Acuña, J.M. (1997); páginas 34 y 35. La cursiva indica la opinión de Anthony.

${ }^{8}$ Lazzati, Santiago C. (1974).

${ }^{9}$ Lazzati, Santiago C.; op. cit.; pág. 11.
} 
Los estados contables son un modelo representativo de la realidad patrimonial de un ente. Como todo modelo es una simplificación que recepta las variables más relevantes y desecha las que no son significativas. Existen reglas para su composición, como en cualquier modelo, que en este caso serían los requisitos de la información contable, entre los que se encuentra la objetividad, según lo veremos.

Lo que debe tenerse siempre presente es que un modelo debe respetar en todo momento el objetivo de su creación. Si el modelo se vuelve un fin en sí mismo, en lugar de un medio para poder tomar decisiones correctas, se tergiversa el objetivo buscado con el mismo. Si por respetar en forma incondicional los principios rectores de la elaboración de un modelo nos alejamos del objetivo que el mismo tiene, estaríamos errando el camino.

Es por esto que el principio de objetividad, como ocurre con todos los demás requisitos de la información contable, no es absoluto sino relativo. Si se omite en los estados contables información relevante para la toma de decisiones en aras de la objetividad, tal vez sea más conveniente flexibilizar un poco el principio en cuestión para incluir aquellos aspectos significativos en la realidad económico-financiera del ente en sus estados contables.

Como veremos a continuación, por cumplir el requisito de objetividad se puede llegar a estar vulnerando otro principio de similar importancia, cual es el de relevancia.

En España, los requisitos de la información contable, enumerados por la Asociación Española de Contabilidad y Administración de Empresas, son:

- Identificabilidad: la información contable debe referirse a sujetos económicos perfectamente definidos en cuanto a su área de actividad económica y al período o momentos que abarca.

- Oportunidad: el momento en que se presenta la información contable a sus usuarios debe ser oportuno para la toma de sus decisiones. Información tardía sería información inútil.

- Claridad: la información contable debe ser clara y asequible, para que pueda ser correctamente interpretada.

- Relevancia: la utilidad de la información contable debe ser notoria para los fines de sus usuarios, completa y pertinente.

- Razonabilidad: dada la imposibilidad práctica de que la información contable refleje a la perfección la situación económica y financiera de una empresa en un momento y/o período determinado, se requiere que la misma refleje con razonabilidad dicha realidad.

- Economicidad: la elaboración de informes contables presupone un costo, pero éste no puede ser superior a los beneficios que de su utilización se obtengan.

- Imparcialidad: la información debe ser neutral, es decir, no debe ser tergiversada a fin de beneficiar y/o perjudicar a determinados usuarios.

- Objetividad: habrá objetividad si varios observadores independientes, aplicando las mismas reglas, arriban a conclusiones similares. 
- Verificabilidad: se requiere que los datos contenidos en los informes contables puedan ser verificados con la respectiva documentación respaldatoria.

- Imagen Fiel: lo cual es corolario de lo anteriormente expuesto.

\section{RECONOCIMIENTO CONTABLE DE ACTIVOS INTANGIBLES: DOCTRINA Y NORMATIVA}

El Plan General de Contabilidad (PGC) de España anterior a la reforma de 2007 no contenía una definición de activos intangibles, sino que simplemente detallaba los conceptos que se incluían como "Inmovilizado inmaterial". El nuevo PGC no subsanó la ausencia de definición, sin embargo, la supliremos a través de distintas normas nacionales e internacionales.

El nuevo PGC define a los activos diciendo que son los "bienes, derechos y otros recursos controlados económicamente por la empresa resultantes de sucesos pasados, de los que se espera que la empresa obtenga beneficios o rendimientos económicos en el futuro".

Intentaremos conceptualizar a los activos intangibles diciendo que son aquellos activos que, además de ser inmateriales, se emplean en forma continua en la actividad principal del ente, tienen una capacidad de servicio que no se agota con el primer empleo, no se transforman en otros bienes al ser utilizados y no se destinan a la venta.

Entre los conceptos que pueden incluirse en el rubro en cuestión encontramos a las patentes de invención, los procesos secretos, las marcas, las concesiones y franquicias, los derechos de uso de software, los derechos de edición y de autor, las investigaciones y desarrollos en proceso, etc.

En cuanto al reconocimiento contable de los intangibles, la doctrina se encuentra dividida. En el ya citado trabajo de Lazzati, el autor distingue entre los valores de uso y de cambio de los activos, siendo susceptible de ser medido objetivamente sólo el segundo. Con posterioridad afirma que los activos tangibles tienen, en su casi totalidad, un valor de cambio que puede ser medido objetivamente, mientras que con los activos intangibles no ocurre siempre lo mismo. Entre ellos separa a los que son identificables como separados del ente y a los que no, siendo sólo los primeros aquellos a los cuales se podría llegar a asignar un valor de cambio objetivo. Es decir, los activos intangibles inidentificables como separados del ente no tienen valor de cambio y, consecuentemente, no pueden ser medidos objetivamente, dejándolos fuera de los estados contables. Ejemplos de este segundo grupo de activos intangibles son la cantidad y fidelidad de la clientela, la especialización y conocimientos de los empleados, la calidad de los bienes y servicios comercializados.

A continuación el autor presenta una crítica, atinada a nuestro criterio, al reconocimiento contable de algunos activos intangibles "inidentificables", ya que suelen asimilarse a los bienes de uso, asignándoles todos los gastos para su desarrollo y luego amortizándolos. Sin embargo, éste dudosamente sea el valor de 
éstos activos, ya que se espera que el resultado de dichos gastos sea más valioso que la inversión efectuada. Por último, presenta al costo histórico como el único dato objetivo para valuar a los "activos intangibles identificables".

Frente a la opinión de Lazzati se encuentra la posición de Fowler Newton.

Éste último autor considera que para que un activo pueda ser considerado contablemente debe tener utilidad económica (es decir, valor de cambio o de uso, sin necesitar un costo asociado), el ente debe poder gozar de los beneficios que el mismo produce basado en un derecho adquirido con anterioridad (sea adquisición, elaboración o por otra vía) y debe poder ser objetivamente valuado.

El único activo intangible que no reúne todos estos requisitos, dice el autor, es el valor llave autogenerado, porque se necesitaría disponer del valor de la empresa y no es frecuente que eso suceda ni que dicho valor sea razonable.

En cuanto al resto de los intangibles, deberían reconocerse contablemente para no subvaluar el patrimonio ni determinar incorrectamente el resultado del ejercicio.

Por último, el autor cita a Harold Bierman y Roland Dukes al rebatir la opinión de muchos autores y de normas contables que consideran apropiado registrar como gastos las erogaciones efectuadas en investigación y desarrollo: "que exista un alto grado de incertidumbre sobre los beneficios futuros de determinados proyectos de investigación y desarrollo, no implica que todos los proyectos deban fracasar, así que es falaz concluir que todos los costos de investigación y desarrollo deben ser cargados a gastos"10.

Además de las posturas doctrinarias antes expuestas, cabe analizar el criterio seguido por las distintas normas contables.

La Norma Internacional de Contabilidad $N^{\circ} 38$ (NIC 38) trata el tema de los activos intangibles. Define, en principio, activo como todo recurso controlado por la entidad como resultado de sucesos pasados y del que la entidad espera obtener beneficios económicos en el futuro. Y estos activos serán intangibles cuando sean identificables, no monetarios y sin apariencia física.

Vale decir que para considerar a una partida como activo intangible, la misma debe ser identificable, lo cual sucede si el mismo es separable o si surge de derechos contractuales o legales.

Una vez cumplidos los requisitos para ser considerado activo, podrá reconocerse contablemente si es probable que los beneficios económicos futuros atribuidos al mismo fluyan a la entidad y si el costo del activo puede valorarse fiablemente.

A éste último respecto, la norma establece que la entidad deberá evaluar la probabilidad de obtener beneficios económicos futuros utilizando hipótesis razonables y fundadas, que representen las mejores estimaciones de la dirección respecto al conjunto de condiciones económicas que existirán durante la vida útil del activo. Asimismo, la entidad utilizará su juicio para evaluar el grado de certidumbre asociado al flujo de beneficios económicos futuros que sea atribuible a la

\footnotetext{
${ }^{10}$ Bierman, H. y Dukes, R.E.; "Accounting for research and development costs”; Journal of Accountancy; abril 1975; citado por Fowler Newton, E. (1995). Pág. 632.
} 
utilización del activo, a partir de la evidencia disponible y otorgando mayor peso a las fuentes externas. Posteriormente se indica que la valuación de los activos intangibles será por su costo.

Recapitulando, podrían resumirse las condiciones para reconocer una partida como activo intangible, según la NIC 38, en las siguientes:

- Recurso controlado por la entidad.

- Se espera obtener beneficios económicos futuros asociados a tal partida.

- Son activos identificables.

- Son activos no monetarios.

- No tienen apariencia física.

- Es probable que los beneficios económicos futuros fluyan al ente.

- Su costo puede valuarse fiablemente.

Ahora bien, si el activo intangible en cuestión se adquirió en forma independiente, los últimos dos requisitos estarían cumplidos, y su valuación estaría dada por el precio de adquisición y todo otro costo directamente atribuible a la preparación del activo para su utilización.

$\mathrm{Si}$, por otro lado, el activo intangible se adquiere en una combinación de negocios, se valuará según su valor razonable a la fecha de adquisición, el cual es el importe por el cual podría ser intercambiado un activo entre partes interesadas y debidamente informadas, en una transacción realizada en condiciones de independencia mutua. En este caso, nuevamente, se considerarán cumplidos los últimos dos requisitos enunciados.

Un tercer caso de introducción de un activo intangible al patrimonio de un ente será la generación interna del mismo ${ }^{11}$. En este caso la NIC 38 establece una discriminación: el fondo de comercio generado internamente no se reconoce como activo y para el resto de los activos intangibles generados internamente deberá distinguirse entre la fase de investigación y la fase de desarrollo. Durante la fase de investigación todas las erogaciones incurridas significarán gastos del ejercicio. Lo mismo ocurrirá en la fase de desarrollo, salvo que se cumplan algunos requisitos que garanticen la finalización de la producción del intangible y su posterior uso o venta, siempre que se puedan valuar fiablemente las erogaciones que necesita.

Es necesario mencionar que en forma explícita la norma sub examine establece que no se reconocerán como activos intangibles las marcas, las listas de clientes, los sellos o denominaciones editoriales u otras similares generadas internamente.

Se concluye, entonces, que los activos intangibles se pueden contabilizar si, además de cumplir todos los requisitos para ser considerados activos e intangibles, se adquieren en forma independiente o en una combinación de negocios (se valuará al costo en el primer caso y al valor razonable en el segundo), mientras que si se

\footnotetext{
${ }^{11}$ La NIC 38 también incluye el caso de adquisición mediante subvención oficial, pero no lo incluiremos en nuestro análisis por considerarlo poco relevante para el fin del presente estudio.
} 
generan internamente, serán considerados gastos, salvo que se cumplan una serie de requisitos y sólo durante la etapa de desarrollo, y se hará, en estos casos, a su costo.

Después del reconocimiento inicial, los activos intangibles podrán ser valuados, en lo sucesivo, a su costo o mediante revalorización. En el primer caso se valuarán a su costo menos las amortizaciones acumuladas y las pérdidas acumuladas por deterioros. En el segundo caso se valuarán a su valor razonable al momento de la revalorización, menos las amortizaciones acumuladas y las pérdidas acumuladas por deterioro.

Por último, consideramos necesario destacar que la importancia que la NIC 38 asigna a los activos intangibles es tal que se destina un capítulo completo a la información a revelar en los estados contables acerca de los mismos. La misma incluye la vida útil asignada a cada intangible (que puede ser finita o indefinida), los métodos de amortización utilizados, los incrementos, amortizaciones y pérdidas por deterioro del valor acaecidas durante el ejercicio, etc. Además se recomienda (aunque no se exige) que se incluya una descripción de los intangibles totalmente amortizados pero todavía en uso y una descripción de los intangibles significativos controlados por la entidad, pero que no se reconozcan como activos por no cumplir con los criterios de reconocimiento fijados en la citada norma.

Ahora bien, analizando la normativa española, el nuevo PGC recoge en gran medida lo establecido por la citada NIC 38.

Para el PGC, es necesario que los intangibles reúnan una serie de condiciones para que puedan ser reconocidos contablemente.

Primeramente, deben ser controlados por la empresa y ser fuente de ingresos futuros, para que pueda considerárselos como activos.

Además, deben poder ser susceptibles de una medición fiable, como los restantes activos y, para el caso particular de los intangibles, deben ser "identificables".

Si una partida reúne todos los requisitos para ser reflejada contablemente como un activo intangible, las formas de valuación que dispone el PGC son, básicamente, el costo de origen depurado de las amortizaciones y el valor razonable, tal como también lo establece la normativa internacional.

$\mathrm{Si}$ los intangibles se adquieren a terceros, se registrarán a su valor de adquisición más los gastos necesarios para ponerlos en uso, al igual que otros activos.

En caso que la incorporación al patrimonio de la empresa de estos activos sea por permutas o aportes de capital no dinerarios, se aplicará el valor razonable para valuarlos. Para el primer caso, sin embargo, se admitirá valuar al intangible incorporado por el valor neto contable del activo entregado, si no fuera posible determinar fiablemente el valor razonable.

También se aplicará el valor razonable cuando se trate de donaciones recibidas.

En el caso de una combinación de negocios, el fondo de comercio, o valor llave, se valuará como la diferencia entre el valor del patrimonio neto de la empresa 
adquirida y el valor razonable de los activos entregados a cambio, siempre y cuando éste procedimiento arroje un valor llave positivo, ya que en caso contrario se registrará una pérdida.

Sólo resta aclarar que no es obligatorio amortizar la llave de negocio en caso que ésta sea positiva, sino que basta con comprobar si la misma ha perdido parte de su valor y registrarlo si así fuera.

Yendo a los casos de intangibles específicos, los gastos de investigación y desarrollo pueden reconocerse como intangibles supeditándose ello al éxito del proyecto y a la clara identificación de los gastos incurridos en el mismo, siendo el plazo máximo de amortización de 5 años.

En cuanto a los derechos sobre activos arrendados, es necesario que la inversión realizada no sea separable del activo arrendado y que incremente su productividad y vida útil, siempre que no pueda ser considerado como un arrendamiento financiero. El plazo de amortización será el menor entre la vida útil de la inversión y la duración del contrato de arrendamiento.

\section{PROPUESTAS DE VALUACIÓN DE ACTIVOS INTANGIBLES CON MATEMÁTICA DIFUSA ${ }^{12}$}

\section{Presentamos aquí al Estado de Valor Estratégico ${ }^{13}$.}

Este estado, que proponemos como adicional o gerencial, expondría la información relevante acerca de los distintos activos intangibles que posee el ente.

De cada activo intangible se informan el valor al cierre y al inicio del período, así como la variación porcentual entre ambas y la participación de cada uno en el total del rubro. Asimismo, se incluye un valor proyectado de cada rubro y la variación que se espera tendrá cada uno durante el ejercicio siguiente. Se incluye, además, la inversión en intangibles realizada durante el ejercicio y su eficiencia.

La composición del cuadro dependerá de la estructura de cada empresa que lo aplique, así como también de la dirección de la misma.

Enunciaremos a continuación algunas formas de valuación de intangibles con Matemática Difusa y las desarrollaremos brevemente.

\footnotetext{
${ }^{12}$ Una versión preliminar de los métodos aquí desarrollados puede encontrarse en Morettini, Mariano (2006) y en Mallo, P.E.et al (2004).

${ }^{13}$ La versión original de este Estado fue presentado con anterioridad en Mallo, P.E. et al (2000).
} 


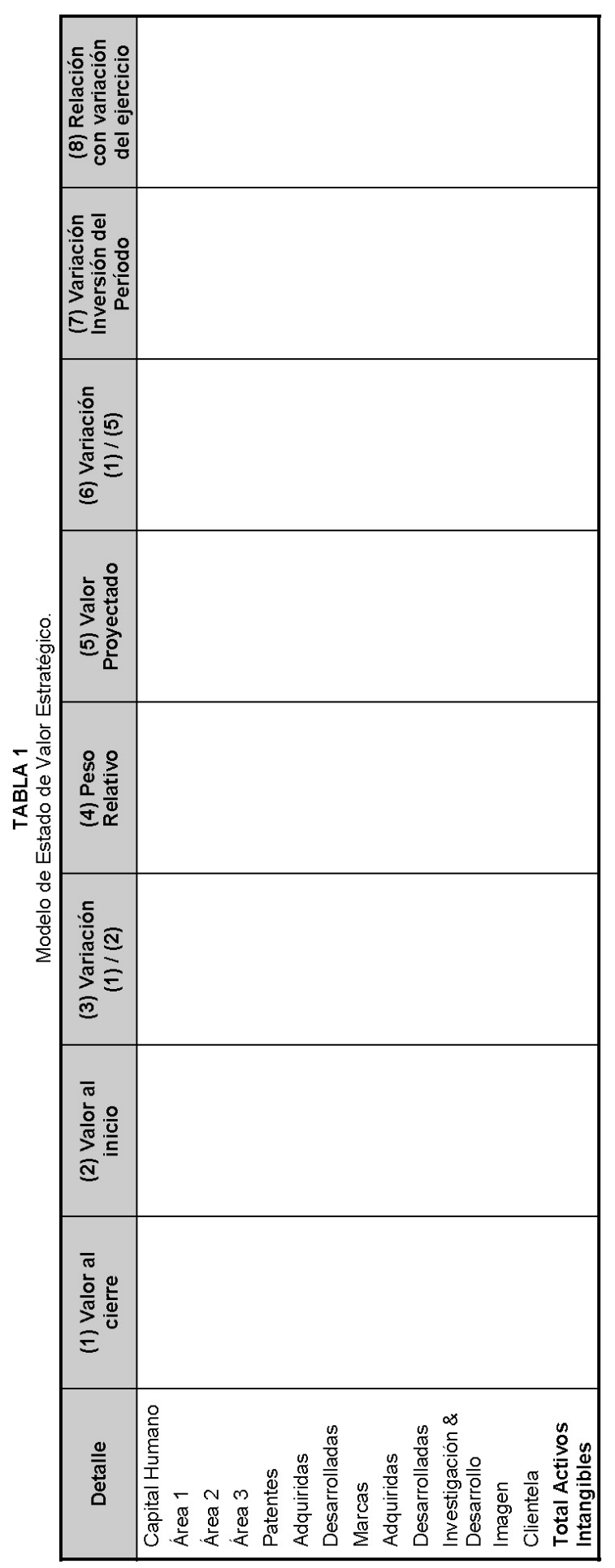

Estudios de Economía Aplicada, 2008: xx-xx • Vol. 26-2 


\subsection{Flujos de fondos descontados}

La primera metodología que presentaremos consiste en aplicar una variante del método del Valor Presente Neto (VPN).

En condiciones de certeza, la fórmula del VPN es la siguiente:

$$
V P N=\sum_{t=0}^{n} \frac{F F N_{t}}{(1+k)^{t}}
$$

donde:

- FFN- $t$ es el flujo de fondos neto correspondiente al período $t$

- $k$ es la tasa de actualización

- $t$ es la medida del tiempo, que puede ser en meses o años, por lo general.

Es evidente que tanto los flujos de fondos netos que se espera obtener en el futuro como la evolución de la tasa de corte en períodos siguientes deberían ser representados por números borrosos, debido a la incertidumbre inherente a dichos valores (el signo $\sim$ encima de cada variable denota que se trata de un número borroso). Introduciendo este concepto, la fórmula anterior quedará:

$$
\tilde{V P N}=\sum_{t=0}^{n} \frac{\tilde{F F} N_{t}}{(1+\tilde{k})^{t}}
$$

La forma de valuar activos intangibles con esta metodología consiste en consultar a un experto en el tema acerca de los flujos de fondos que se espera que generen estos activos en el futuro, a la vez que el mismo u otro experto puede determinar la tasa de corte que debe utilizarse para descontar dichos flujos.

Así, los consumidores adquieren un producto no sólo por sus cualidades intrínsecas, sino también por su marca, la cual se ha posicionado en sus mentes gracias

a la publicidad, al prestigio de la misma a través de acciones de conciencia social llevadas a cabo por la empresa, solvencia técnica de sus desarrolladores, efectividad en la satisfacción de necesidades, adecuada atención al cliente, o cualquier otra cualidad relacionada con la marca que exceda las características objetivas e intrínsecas del bien o servicio ofrecido.

La valuación que deberá hacer el experto de la marca en cuestión consistirá en estimar cuán mayores serían las ventas del producto debido a la marca que éste tiene. Deberá estimarse cuáles serán las ventas del producto en un período futuro determinado - recomendándose un horizonte temporal adecuado - y cuáles serían éstas si el producto careciera de marca o ésta fuera desconocida por los consumidores. La diferencia entre ambas estimaciones puede resultar el valor de la marca, previo descuento por la tasa de corte correspondiente. 
Como puede apreciarse, la complejidad y la incertidumbre inherente en este tipo de tareas amerita la utilización de la Matemática Borrosa, constituyéndose así en una metodología acorde a la situación planteada.

Se presenta aquí la situación de un sólo un experto consultado, pero lo ideal sería consultar a varios y agregar las opiniones según lo expondremos luego.

Para el caso de la valuación de marcas, por ejemplo, los distintos expertos a quienes se puede consultar, entre otros, son el Gerente de Comercialización de la empresa, Consultores en Marketing, Consultoras en Benchmarking y Agencias de Publicidad y Posicionamiento de marcas.

La forma en que los expertos arriban a sus estimaciones es, en principio, irrelevante. No obstante ello, indicaremos que no solo la experiencia y el conocimiento personal sobre el tema son determinantes a la hora de la valuación de estos activos, sino la utilización de herramientas estadísticas, matemáticas, económicas, financieras u otras pertinentes a la tarea son de suma importancia.

\subsection{Agregación de opinión de expertos}

La segunda alternativa que presentaremos aquí ${ }^{14}$ consiste en utilizar alguna técnica para la agregación de la opinión de expertos - en tanto estas fueran cuantitativas, como es el presente caso, en el que se trata de cuantificar el valor de los intangibles - . Trataremos la metodología Fuzzy-Delphi, donde se obtiene un valor que represente a todas las opiniones recabadas, pero siguiendo una metodología particular, cual es la denominada Delphi.

El método Delphi consiste en pedir la opinión anónima a distintos expertos sobre algún hecho o situación incierta. Las opiniones se procesan, calculándose una mediana y recorrido intercuartílico, y se reformula la pregunta a cada experto, informando las medidas de la primera ronda de respuestas, para que cada experto reformule, si lo desea, su opinión.

Supóngase que se pretende utilizar esta forma de agregación de opiniones para la valuación de la fidelidad de la clientela, y se pide la opinión de diferentes expertos sobre el tema, que pueden ser tanto internos como externos a la em$\operatorname{presa}^{15}$.

La forma en que tales expertos arriben a sus resultados es, repetimos, irrelevante. Sólo debería preguntarse entre qué montos mínimo y máximo cree que se encuentra el valor de la clientela de la empresa, considerando su fidelidad, y, adicionalmente, pedirle que indique un monto, dentro del intervalo propuesto, que considere como más representativo de dicho valor.

\footnotetext{
${ }^{14}$ No es el objetivo del presente trabajo desarrollar demasiadas técnicas de valuación con herramientas difusas, sino sólo presentar algunas para evaluar la necesidad de las mismas y su adaptación a los criterios contables.

${ }^{15}$ Para ver otras aplicaciones así como un caso de aplicación de mayor complejidad, puede cónsultarse Gil Lafuente, A.M. (1990).
} 
A partir de las opiniones puede trazarse el número borroso medio, cuyos valores característicos son el promedio de los valores característicos de los NBTs considerados.

Informando cuál fue el NBT medio y la distancia al mismo a cada experto, pueden efectuarse sucesivas rondas para que cada uno revea su opinión. Si luego de algunas rondas hay opiniones que siguen quedando alejadas del NBT medio, conviene analizar las causas.

Una vez finalizado el proceso, se toma el NBT medio como estimación definitiva del valor de los activos intangibles analizados, aunque existen otras posturas, como la experiencia PERT, de dar mayor ponderación (4/6 por ejemplo) al valor central y menor peso $(1 / 6)$ a los valores extremos.

FIGURA 3

Opiniones de expertos y NBT medio.

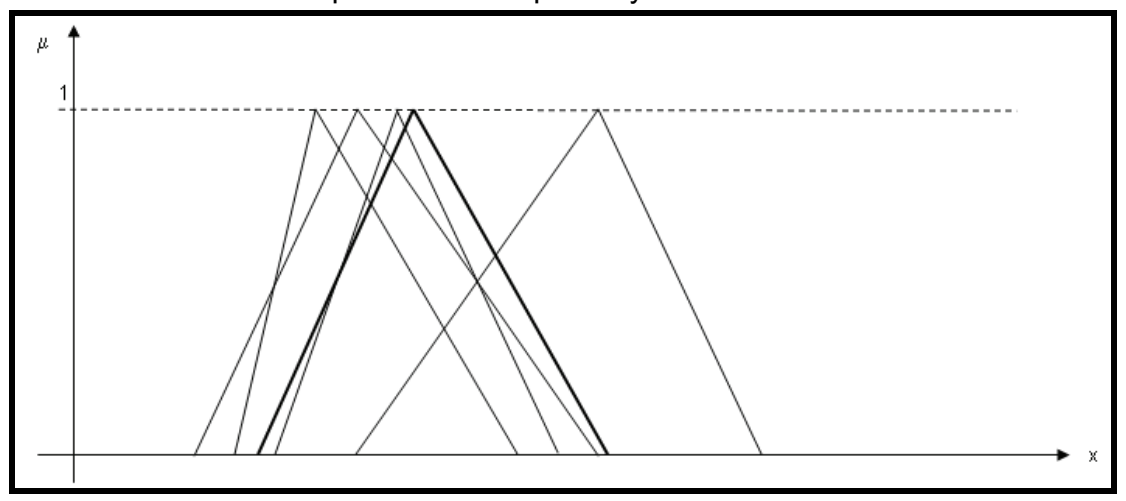

En la Figura 3 se muestra el gráfico de cuatro opiniones de expertos acerca del valor de un activo intangible y el NBT medio resultante, cuyo trazo es más grueso que el resto. Puede observarse que uno de los expertos está claramente valuando al intangible en cuestión por encima de lo que consideran las otras tres personas consultadas. Al informarse el NBT medio, éste puede revisar su opinión y acercar una nueva estimación, siguiendo con el proceso de la manera ya descripta.

Cabe aclarar que las mencionadas son sólo algunas de las técnicas basadas en la lógica difusa para agregar opiniones de expertos, habiendo una vasta cantidad de técnicas adicionales, que por ser más complejas, y en aras de la didáctica, no mencionaremos en este trabajo.

\section{ADAPTACIÓN A LOS REQUISITOS DE LA INFORMACIÓN CONTABLE}

Una vez presentadas algunas de las alternativas para la valuación de activos intangibles con Matemática Difusa, corresponde analizar si de esta manera se 
cumple con los requisitos de la información contable y si se contribuye al mejor cumplimiento de los objetivos de los estados contables.

Claramente, la normativa contable vigente adopta criterios de reconocimiento y valuación de los activos intangibles que atentan contra el objetivo de los estados contables.

Con la introducción de la Matemática Difusa para valuar los activos intangibles, se sincera la información a brindar a los usuarios de los estados contables, en el sentido que el resultado no tendrá la precisión de la Matemática tradicional, pero será coherente con la dificultad implícita en la valuación de dichos activos y otorgará un rango de valores que representará el valor de los intangibles.

Quien tenga que tomar decisiones de distinta índole frente a los estados contables de un ente, necesitará que la información contenida en ellos sea lo más fiel posible a la realidad económico-financiera del mismo. Con la normativa vigente es evidente que ello no ocurre, por lo menos en cuanto a los activos intangibles se refiere. Consideramos que es preferible rescindir precisión en la valuación de los mismos a exactitud. Indiscutiblemente es más preciso informar que el valor de los bienes intangibles de un ente es de $€ 0,00$ que decir que es de entre $€ 80.000,00$ y $€ 130.000,00$, siendo el valor más confiable $€ 100.000,00$, pero también es indiscutible que el primero está mucho más reñido con la realidad que el segundo.

Debido a la falta de precisión de los números borrosos triangulares que se expondrían en el Estado de Valor Estratégico, consideramos pertinente incluir al mismo como información complementaria a los Estados Contables, e incluir en éstos el promedio de los tres valores característicos.

Además, el valor determinado por medio de las técnicas borrosas, que estarán incluídos en el Estado de Valor Estratégico, podrá servir como valor límite de los activos intangibles, en caso que el valor contable de los mismos continúe informándose como hasta ahora y no se adopten nuestras propuestas.

Así, ningún activo intangible podrá tener un valor reflejado en los estados contables que sea superior al límite máximo del número borroso triangular que le corresponda en el Estado de Valor Estratégico.

Si consideramos los cuatro aspectos fundamentales en los que son útiles los estados contables según Juan Carlos Viegas, vemos que la utilidad de las técnicas borrosas es mayor que las prácticas actuales.

En relación al primer aspecto, el control de gestión, lo más importante es vincular el resultado del ejercicio con la inversión del período, a fin de determinar la rentabilidad de la misma. Indudablemente, si un programa de capacitación al personal cuesta $€ 20.000$ pero se estima que redundará en una disminución de costos de entre $€ 60.000$ y $€ 80.000$, con máxima confianza en $€ 75.000$, no será lo mismo informar que los $€ 20.000$ invertidos son pérdida que informar que se logró incrementar las capacidades del personal aumentando el valor de los activos intangibles. La información contable actual, según la normativa vigente, informaría que la decisión del ente de encarar dicha capacitación perjudicó la rentabilidad de 
la empresa e incrementó los gastos, lo cual es falso, ya que de ser así nunca se hubiera tomado esa decisión. El Estado de Valor Estratégico informaría, en cambio, que se logró mejorar la competitividad de la fuerza laboral esperando resultados positivos de entre 3 y 4 veces la inversión efectuada.

En cuanto a la financiación de la empresa, no se logran mejores resultados con la introducción de la Matemática Difusa considerando el corto plazo, pero al pensar en el largo plazo sí, porque entran en juego los estados contables proyectados, donde el papel de las técnicas borrosas para la valuación de los intangibles, entre otros, es fundamental.

Respecto del valor de la empresa, es evidente el aporte de la Matemática Difusa. No es lo mismo decir que el ente no posee activos intangibles (lo que equivale

a no reconocerlos contablemente) que decir que existen y dar su valor aproximado. En éste último caso el Estado de Valor Estratégico es crucial, y el aporte de la Matemática Difusa, esencial en su elaboración.

En cuanto al último aspecto, el saneamiento financiero, es limitado el aporte de los estados contables, y también el de la introducción de nuestras propuestas.

Veamos ahora si las técnicas presentadas cumplen con los requisitos de la información contable, según la normativa española.

- Identificabilidad: nuestras propuestas no modifican en absoluto el cumplimiento de este requisito.

- Oportunidad: la aplicación de las técnicas que proponemos evidentemente acarrearía mayor trabajo y tiempo que informar el valor de los intangibles como nulo, pero ese mayor tiempo no es significativo como para atentar contra el requisito de oportunidad.

- Claridad: no se afecta este principio por la introducción de técnicas borrosas.

- Relevancia: con las actuales normas se está obviando la presentación de información muy relevante para sus usuarios. La introducción de nuestras propuestas incorporaría dicha información relevante, esto es, la valuación de los distintos intangibles de la empresa. Además, al brindar una valuación de los activos intangibles acordes a la realidad, permiten confirmar si las inversiones efectuadas en los mismos fueron fructíferas o no, y ayudan a mejorar los pronósticos. Por otra parte, se reconocería todo activo intangible en función de la realidad económica, a diferencia de las reglas actuales de reconocimiento de intangibles. Por último, si alguna técnica para valuar intangibles no cumple con el requisito de que la información sea completa es la contemplada en las normas contables vigentes: nuestra propuesta reconocería todos los activos intangibles, cumpliendo con este requisito más acabadamente.

- Razonabilidad: si mediante la aplicación de las normas contables vigentes se logra reflejar razonablemente la situación económica-financiera de una empresa, con nuestra propuesta dicha razonabilidad será aún mayor, porque reflejará mejor el valor de activos que ahora están subvaluados o ignorados. 
- Economicidad: la medición de los intangibles mediante nuestras propuestas no tienen por qué encarecer los trabajos contables tanto como para que deje de cumplirse con el principio de economicidad.

- Imparcialidad: la correcta aplicación de las técnicas borrosas no tienen por qué beneficiar a algunos usuarios en detrimento de otros, ya que se trata simplemente de una técnica alternativa, por lo que la imparcialidad estaría, en todo caso, en quien las aplica.

- Objetividad: tal vez la información brindada con técnicas borrosas no sea lo objetiva que es la información actual. Una alternativa para mejorar esto sería consultar a distintos expertos en cada tema para luego obtener una opinión representativa de todos, es decir, aplicar el método Fuzzy-Delphi, por ejemplo. Tampoco hay que confundir objetividad con precisión. Con las metodologías presentadas puede arribarse a valores objetivos en el sentido que dos observadores independientes pueden alcanzar resultados similares, aunque estos no sean precisos sino números borrosos.

- Verificabilidad: consultando a distintos expertos para valuar un intangible se logra este requisito.

\section{CONCLUSIONES}

En la actualidad, la importancia de los activos intangibles para el éxito de cualquier negocio es vital y creciente. Independientemente de si son o no identificables como separados del ente, fueron adquiridos o autogenerados o su valuación es fácilmente realizable o no, es indudable que son activos, y muy importantes, y como tales deben ser reflejados contablemente.

No hacerlo significaría subvaluar el activo y determinar incorrectamente el resultado del ejercicio, brindando, en consecuencia, información incorrecta a los usuarios de los estados contables para la toma de decisiones.

El problema radica, entonces, en la forma de valuar a dichos intangibles. Las normas contables vigentes han preferido preservar la prudencia y objetividad en detrimento de los objetivos de los estados contables, al no reconocer contablemente a los intangibles autogenerados (salvo escasas excepciones contempladas en las normas internacionales de contabilidad, como se expuso en su momento).

Nuestra propuesta consiste en presentar un Estado de Valor Estratégico complementario de los estados contables básicos, que brinde información acabada sobre los distintos activos intangibles de manera que se constituya en información útil y veraz sobre la realidad económica-financiera del ente y puedan así sus usuarios enfrentar la toma de decisiones mejor informados.

La valuación de los intangibles que contendría dicho estado se haría utilizando la Matemática Borrosa. De esta manera no sólo se cumplen todos los requisitos 
que debe contener la información contable según la normativa vigente, sino que mejora la utilidad de los estados contables en su conjunto.

Según las normas contables vigentes, la gran mayoría de los intangibles tendrá valores contables nulos, y los restantes tendrán valores contables menores a los reales, ya que deberá elegirse el menor entre: a) Costo neto de amortizaciones acumuladas; b) Valor si se vende; y c) Valor si se utiliza.

Consideramos que si se quiere mantener un conservadurismo tal en la información a brindar en los estados contables, es vital la inclusión de información complementaria informando sobre los valores reales de estos activos ${ }^{16}$. El Estado de Valor Estratégico cubriría este déficit.

\section{REFERENCIAS BIBLIOGRÁFICAS}

CAÑIBANO CALVO, L. y GISBERT CLEMENTE, A. (2007): "Los activos intangibles en el nuevo Plan General Contable", 2007, "El nuevo Plan General Contable", Revista del Instituto de Estudios Económicos, $\mathrm{n}^{\circ}$ 2-3.

Federación Argentina de Graduados en Ciencias Económicas, Instituto Técnico de Contadores Públicos (2004): "Activos Intangibles", Anales del $15^{\circ}$ Congreso Nacional de Profesionales en Ciencias Económicas, Salta.

FOWLER NEWTON, ENRIQUE (1998): Contabilidad Superior, Ediciones Macchi, Buenos Aires.

FOWLER NEWTON, ENRIQUE (2005): Cuestiones Contables Fundamentales, $4^{\circ}$ edición, La Ley, Buenos Aires.

GIL LAFUENTE, A.M. (1990): El análisis financiero en la incertidumbre, Ariel, Barcelona.

International Accounting Standards Comitee; IAS 38

KAUFMANN, ARNOLD y GIL ALUJA, JAUME (1987): Técnicas operativas de gestión para el tratamiento de la incertidumbre, Editorial Hispano Europea, Barcelona.

KOSKO, BART (1995): Pensamiento borroso, Editorial Crítica.

LAZZARI, L.L.; MACHADO, E.A.M. y PÉREZ, R.H. (1998): Teoría de la Decisión Fuzzy, Ediciones Macchi, Buenos Aires.

LAZZATI, SANTIAGO C. (1974): El objetivo de los Estados Contables, Ediciones Macchi, Buenos Aires.

MALLO, P.E. et al (2000): "El Estado de Valor Estratégico", Anales del VIl Congreso de la Sociedad Internacional de Gestión y Economía Fuzzy, Creta, Grecia.

MALLO, P.E. et al (2004): "Valuación de Empresas con Técnicas Borrosas", Anales del $15^{\circ}$ Congreso Nacional de Profesionales en Ciencias Económicas, Salta, Argentina.

MALLO, P.E.; ARTOLA, M.A.; PASCUAL, M.E.; GARCÍA, M.V. y MARTINEZZ, D. (2004): Gestión de la incertidumbre en los negocios, RIL Editores, Editorial Melusina, Santiago de Chile.

MORETTINI, MARIANO (2006): "Valuación de Activos Intangibles en torno a los objetivos de los estados contables: una propuesta de cambio", Trabajo galardonado con el Premio Jóvenes Profesionales de la Federación Argentina de Consejos Profesionales de Ciencias Económicas, Edición 2006.

Real Decreto 1514/2007 del Reino de España.

VIEGAS, J.C.; FRONTI, L.; CHAVES, O.A. y PAHLEN ACUÑA, J.M. (1997): Contabilidad: Presente y Futuro, Ediciones Macchi, Buenos Aires.

\footnotetext{
${ }^{16}$ Iguales consideraciones valen para el resto de los activos, aunque nos reservamos su análisis para otra ocasión, ya que escapan al objetivo de este trabajo.
} 
ZADEH, LOFTI A. (1965): “Fuzzy Sets”, Inform. Control, vol. 8; pp. 338-353. 